Asian J. Med. Biol. Res. 2017, 3 (4), 534-538; doi: 10.3329/ajmbr.v3i4.35346

\author{
Asian Journal of \\ Medical and Biological Research \\ ISSN 2411-4472 (Print) 2412-5571 (Online) \\ www.ebupress.com/journal/ajmbr
}

\title{
Short Communication \\ Antibiotic resistance pattern of Salmonella spp. isolated from stool samples of hospitalized diarrheal patients in Bangladesh
}

\author{
Mohammad Sharif Uddin ${ }^{1}$, Md. Imranul Hoq ${ }^{1}$, Mohammad Shaokat Ali ${ }^{2 *}$, Md. Mijanur Rahman ${ }^{1}$ and K. M. \\ Shariful Islam ${ }^{1}$ \\ ${ }^{1}$ Department of Microbiology, University of Chittagong, Bangladesh \\ ${ }^{2}$ Department of Applied Chemistry and Chemical Technology, Faculty of Food Science and Technology, \\ Chittagong Veterinary and Animal Sciences University, Khulshi, Chittagong-4225, Bangladesh
}

*Corresponding author: Mohammad Shaokat Ali, Department of Applied Chemistry and Chemical Technology, Faculty of Food Science and Technology, Chittagong Veterinary and Animal Sciences University, Khulshi, Chittagong-4225. Phone: +8801825128942; E-mail: shaokat.fst@gmail.com

Received: 07 December 2017/Accepted: 20 December 2017/ Published: 28 December 2017

\begin{abstract}
This study was carried out to investigate the drug resistance pattern of Salmonella spp. from diarrheal patients of under 5 years of age. Collected stool samples from the hospitalized diarrheal patients were analyzed for the presence of Salmonella spp. Among 350 stool samples from diarrheal patients, 15 (4\%) were positive for the Salmonella spp. Antibiotic susceptibility test of the identified Salmonella spp. was performed according to Kirby-Bauer disc diffusion method. Eight commonly used antibiotics including azithromycin $(15 \mu \mathrm{g})$, chloramphenicol $(30 \mu \mathrm{g})$, Sulphamethaxole-trimethoprim $(25 \mu \mathrm{g})$, metronidazole $(50 \mu \mathrm{g})$, tetracycline $(30 \mu \mathrm{g})$, doxycycline $(30 \mu \mathrm{g})$, erythromycin $(15 \mu \mathrm{g})$ and ciprofloxacin $(5 \mu \mathrm{g})$ used to determine drug resistance pattern of the identified Salmonella spp. Majority of the isolates were multidrug resistant showed resistance against more than three drugs. $73 \%$ of Salmonella spp. was resistant to 2-4 drugs and 20\% showed resistance to more than 5 drugs. We found that $100 \%$ Salmonella showed resistance to metronidazole and $87 \%$ were resistant to erythromycin. $7 \%$ isolates were resistant to chloramphenicol and doxycycline and $20 \%$ were resistant to ciprofloxacin. Antibiotic resistance is a serious public health problem worldwide and a leading cause of mortality and morbidity. This kind of situation, leads to great socioeconomic losses from the perspective of the patient, the hospital, and the whole society. To get rid form the drug resistance problem unnecessary, over and misuse of antibiotics should immediately prohibited and policy of judicious use of antibiotics should be strengthened.
\end{abstract}

Keywords: hospitalized diarrheal patients; stool samples; Salmonella spp.; antibiotic resistance pattern

\footnotetext{
1. Introduction

Salmonellosis is a growing concern day by day throughout the world and over the last several decades there have been significant shift in predominant. Salmonella serovars are associated with human infections. Nontyphoidal Salmonella serovars cause as much as an estimated 1 billion cases of gastroenteritis in humans every year (Kennedy et al., 2004; Coburn et al., 2007). Furthermore, they are also responsible for some serious infections such as septicemia and endocarditis (Gill, 1979), empyema (Burney et al., 1977), and meningitis (Chusid et al., 1980), especially in immunocompromised host. Although most Salmonella infections are selflimiting, effective antimicrobial therapy is needed if spread beyond the intestine occurs. The progressive increase in antimicrobial resistance in enteric pathogens is of greatest concern in the developing world. Since 1960s, several Salmonella spp. have been showed resistance to various common antibiotics such as ampicillin, chloramphenicol, and sulfamethoxazole-trimethoprim with increasing frequency throughout the world (Smith $e t$ al., 1984). Extended-spectrum cephalosporins and fluoroquinolones have become the drugs of choice for the
} 
treatment of infections caused by multidrug-resistant Salmonella serotypes due to the increased resistance to conventional antibiotics. However, since 1991, several studies from different countries have been reporting cases of infections by extended-spectrum cephalosporins resistant salmonellae (Dunne et al., 2000; Fey et al., 2000). According to a study by Gupta et al., in the United States, from 1998 through 2001, a 5-fold increase in the prevalence of Salmonella species resistant to expanded-spectrum cephalosporins (Gupta et al., 2003). In 10 European countries a survey conducted in 2000 identified a cefotaxime resistance rate of $0.6 \%$ in Salmonella isolates recovered from human sources (Threlfall et al., 2000). In recent years, such a trend of increase in drugresistant salmonellae including resistance to quinolones has been observed in many countries, particularly in Asia (Lauderdale et al., 2006; Wang et al., 2006). The use of antimicrobials in any purpose, including disease treatment and growth promotion in domestic livestock, can potentially lead to develop drug resistant bacteria. (Gomez-Lus, 1998; Tollefson et al., 1997). Mechanisms of bacterial antimicrobial resistance includes (i) changes in bacterial cell wall permeability, (ii) removal of antimicrobials via efflux pumps mechanisms (iii) modification of the site of drug action, and (iv) destruction or inactivation of antimicrobials (Barbosa and Levy, 2000; Schwarz and Chaslus-Dancla 2001). Most often, acquired antimicrobial resistance phenotypes develop by the transfer of plasmids via conjugation process (Guerra et al., 2002; Gebreyes and Altier, 2002). Plasmids may carry class I integrons, which are mobile DNA elements, play a vital role in the proliferation of bacterial multidrug resistance (MDR) (Arduino et al., 2002; Di Conza et al., 2002). We have conducted this study on diarrheal patients of less than five years of age in a private hospital of Chittagong district of Bangladesh.

\section{Materials and Methods}

\subsection{Sample Collection}

A total 350 fresh watery stool samples from patients of less than 5 years exhibiting symptoms of gastroenteritis were collected from different hospitals of Chittagong, Bangladesh. Then the collected samples were taken in frozen refrigerant packs and transported to the laboratory immediately.

\subsection{Isolation and identification of Salmonella spp.}

Collected samples were inoculated in Luria-Bertani (LB) broth for the enrichment of bacteria and incubated at $37^{\circ} \mathrm{C}$ for 24 hours. After overnigt of enrichment, cultures were aseptically inoculated onto Hektoen enteric agar and Salmonella-Shigella (SS) agar (Oxoid, Hampshire, England) plates and incubated at $37^{\circ} \mathrm{C}$ for $24 \mathrm{~h}$. Then the Hektoen agar plates were examined for characteristic green or blue-green colonies (with or without black centre), and distinct colorless colonies onto Salmonella-Shigella (SS) agar plates were primarily selected as species of Salmonella. After primary confirmation each isolates were finally confirmed as Salmonella by conducting several physiological and biochemical tests. Motile isolates from primary selection with negative indole tests where red violet colored ring in the top of the broth cultures after adding kovac's solution didn't formed, positive citrate tests where blue green color of the media formed and, provided red alkaline slant and yellow acidic butt with black colored $\mathrm{H}_{2} \mathrm{~S}$ production were confirmed as Salmonella.

\subsection{Antimicrobial susceptibility test}

Antibiotics susceptibility test was performed by Kirby-Bauer disc diffusion method (Hudzicki, 2009). Bacterial suspensions were prepared from fresh culture grown overnight onto nutrient agar plates by using sterile normal saline and the turbidity of the suspension was adjusted to $0.5 \mathrm{McFarland}$ Standard that corresponds to approximately $1 \times 10^{8} \mathrm{CFU} / \mathrm{ml}$ of suspension. A sterile cotton swab was dipped into the inoculum then streaked on the Mueller-Hinton agar plate properly. Then antibiotic discs impregnated with azithromycin $(15 \mu \mathrm{g})$, chloramphenicol $(30 \mu \mathrm{g})$, Sulphamethaxole-trimethoprim $(25 \mu \mathrm{g})$, metronidazole $(50 \mu \mathrm{g})$, tetracycline $(30 \mu \mathrm{g})$, doxycycline $(30 \mu \mathrm{g})$, erythromycin $(15 \mu \mathrm{g})$, ciprofloxacin $(5 \mu \mathrm{g})$ (Oxoid, UK) were dispensed onto the dried agar surface using a sterile forceps. The plates were incubated overnight at $37^{\circ} \mathrm{C}$. After incubation period, the resulted zone of inhibition was compared with that of CLSI guideline (CLSI, 2010) for the interpretation of the data and categorization of the test strains as intermediate, sensitive, or resistant.

\section{Results and Discussion}

Among 350 collected samples from diarrheal patients, 4\% (15) of them were positive for Salmonella spp. Results of antibiotic resistance pattern of the isolated Salmonella spp. are shown in Table 1 and Figure 1. Majority of the isolates were multidrug resistant having resistance pattern against at least three antibiotics. We found that $100 \%$ (15) of Salmonella spp. showed resistance to metronidazole and 87\% (13) were resistant to erythromycin. $7 \%$ (1) isolates were resistant to chloramphenicol and doxycycline and 20\% (3) were resistant to 
ciprofloxacin. $40 \%$ (6) of the total isolates were resistant to azithromycin and trimethoprim-sulfamethoxazole and $33 \%$ (4) were resistant to tetracycline.

In developing countries multidrug resistance in bacteria is a common problem including South Asia. This problem is most likely related to the frequent use of antibiotics without proper medical supervision (Sack et al., 1997).

The goal of this study was to investigate drug resistance patterns of the identified Salmonella spp. in Bangladesh. This study results revealed that $20 \%$ Salmonella were resistant to more than 5 drugs and $73 \%$ were resistant to 2-4 drugs. In another result we found that $20 \%$ Salmonella was resistant to ciprofloxacin which is similar to the study conducted by Xia et al., 2009. Reports from other countries have found an increasing frequency of ciprofloxacin-resistant isolates (Cailhol et al., 2006; Davis et al., 1999). Ciprofloxacin-resistant isolates are involved with increased morbidity and mortality (Varma et al., 2005; Helms et al., 2004) and high frequency makes it difficult to use fluoroquinolones for treatment of Salmonella infections in humans. Due to the increasing rate of antimicrobial resistance among salmonellae, broad-spectrum agents such as imipenem or cefepime are now more likely to be used for treatment. These antibiotics are usually more expensive and toxic, and more harmful to the commensurate microflora. This kind of situation, leads to great socioeconomic losses from the perspective of the patient, the hospital, and the whole society. A recent report stated that, they have isolated a Salmonella strain that was resistant to imipenem, which is a most fearful indication for future (Miriagou et al., 2003).

Table 1. Susceptibility of Salmonella isolates to various antimicrobial agents.

\begin{tabular}{llll}
\hline Antibiotics & \multicolumn{2}{c}{ Number of isolates } \\
\cline { 2 - 4 } & Resistant & Intermediate & Sensitive \\
\hline Azithromycin & 6 & 4 & 5 \\
Chloramphenicol & 1 & 0 & 14 \\
Sulfamethoxazole-trimethoprim & 6 & 1 & 8 \\
Metronidazole & 14 & 1 & 0 \\
Tetracycline & 4 & 3 & 8 \\
Doxycycline & 1 & 6 & 8 \\
Erythromycin & 13 & 1 & 1 \\
Ciprofloxacin & 3 & 5 & 7 \\
\hline
\end{tabular}

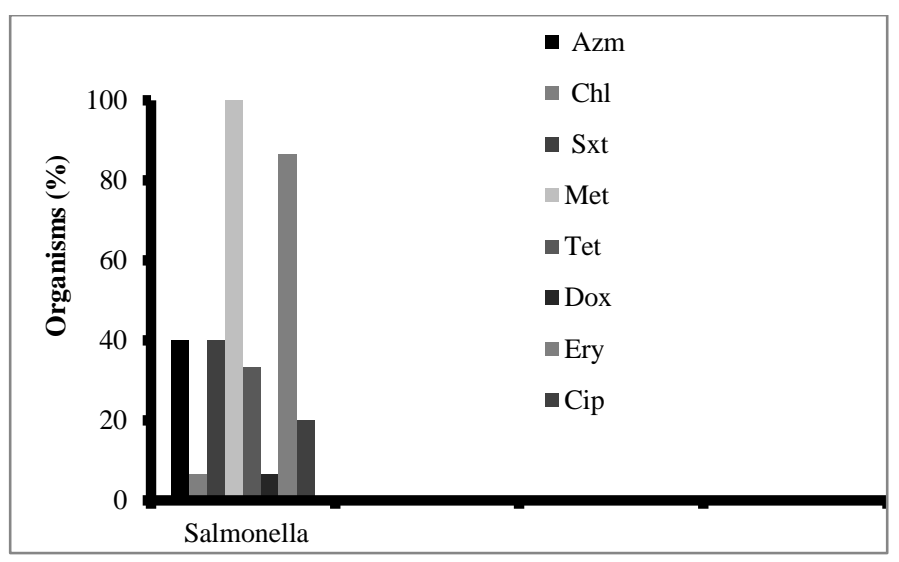

Figure 1. Distribution of isolates (Salmonella) according to antibiotic resistance pattern.

Note: Azm= Azithromycin, $\mathrm{Chl}=$ Chloramphenicol, $\mathrm{Sxt}=$ Sulfamethoxazole-trimethoprim, Met= Metronidazole,

Tet $=$ Tetracycline, Dox $=$ Doxycycline, Ery= Erythromycin, Cip= Ciprofloxacin

\section{Conclusions}

Drug resistant Salmonella have constituted a serious threat to public health. To overcome the challenge of drug resistance of Salmonellae, overuse and misuse of antimicrobial agents in food animals should be stopped (McEwen et al., 2002; Swartz et al., 2002). Continued surveillance for resistance pattern for salmonellae is mandatory and ongoing surveillance should be expanded and well standardized. It is however expected that this study will open a new window in exploring the true condition of antibiotic resistance patterns of Salmonella in Bangladesh. 


\section{Acknowledgements}

The authors would gratefully like to acknowledge the laboratory support given by the Bangladesh Council of Scientific and Industrial Research (BCSIR), Chittagong.

\section{Conflict of interest}

None to declare.

\section{Reference}

Arduino SM, PH Roy, GA Jacoby, BE Orman, SA Pineiro and D Centron, 2002. blaCTX-M-2 is located in an unusual class 1 integron (In35) which includes Orf513. Antimicrob. Agents Chemother., 46: 2303-2306.

Barbosa TM and SB Levy, 2000. The impact of antibiotic use on resistance development and persistence. Drug Resist Update, 3: 303-311.

Burney DP, RD Fisher and W Schaffner, 1977. Salmonella empyema: a review. South. Med. J., 70: 375-377.

Cailhol J, R Lailler, P Bouvet, S La Vieille, F Gauchard, P Sanders and A Brisabois, 2006. Trends in antimicrobial resistance phenotypes in nontyphoid salmonellae from human and poultry origins in France. Epidemiol. Infect., 134: 171-178.

Chusid MJ, TH Duningan and DS Lewis, 1980. Salmonella meningitis in infancy. Wis. Med. J., 79: 23-25.

CLSI, 2010. Performance standards for antimicrobial susceptibility testing; Seventeenth informational supplement. M100-S17, Wayne, PA.

Davis MA, DD Hancock, TE Besser, D H Rice, JM Gay, C Gay, L Gearhart and R DiGiacomo, 1999. Changes in antimicrobial resistance among Salmonella enterica serovar Typhimurium isolates from humans and cattle in the Northwestern United States, 1982-1997. Emerg. Infect. Dis., 5: 802-806.

Di Conza J, JA Ayala, P Power, M Mollerach and G Gutkind, 2002. Novel class 1 integron (InS21) carrying blaCTX-M-2 in Salmonella enterica serovar Infantis. Antimicrob. Agents Chemother., 46: 2257-2261.

Dunne EF, PD Fey and P Kludt, 2000. Emergence of domestically acquired ceftriaxone-resistant Salmonella infections associated with AmpC blactamase. JAMA, 284: 3151-6.

Fey PD, TJ Safranek, ME Rupp, EF Dunne, E Ribot, PC Iwen, PA Bradford, FJ Angulo and SH Hinrichs, 2000. Ceftriaxone-resistant Salmonella infection acquired by a child from cattle. N. Engl. J. Med., 342: 1242-9.

Gebreyes WA and C Altier, 2002. Molecular characterization of multidrug-resistant Salmonella enterica subsp. enterica serovar Typhimurium isolates from swine. J. Clin. Microbiol., 40: 2813-2822.

Gill GV, 1979. Endocarditis caused by Salmonella enteritidis. Br. Heart J., 42: 353-354.

Gomez-Lus R, 1998. Evolution of bacterial resistance to antibiotics during the last three decades. Int. Microbiol., 1: 279-284.

Guerra B, S Soto, R Helmuth and MC Mendoza, 2002. Characterization of a self-transferable plasmid from Salmonella enterica serotype Typhimurium clinical isolates carrying two integron-borne gene cassettes together with virulence and drug resistance genes. Antimicrob. Agents Chemother., 46: 2977-2981.

Gupta A, J Fontana and C Crowe, 2003. Emergence of multidrug-resistant Salmonella enterica serotype Newport infections resistant to expandedspectrum cephalosporins in the United States. J. Infect. Dis., 188: $1707-16$.

Helms M, J Simonsen and K Mølbak, 2004. Quinolone resistance is associated with increased risk of invasive illness or death during infection with Salmonella serotype Typhimurium. J. Infect. Dis., 190: 1652-1654.

Hudzicki J, 2009. Kirby-bauer disk diffusion susceptibility test protocol.

Lauderdale TL, FM Aarestrup, PC Chen, JF Lai, HY Wang, YR Shiau, IW Huang and CL Hung, 2006. Multidrug resistance among different serotypes of clinical Salmonella isolates in Taiwan. Diagn. Microbiol. Infect. Dis., 55: 149-155.

McEwen SA and PJ Fedorka-Cray, 2002. Antimicrobial use and resistance in animals. Clin. Infect. Dis., 34: 93106.

Miriagou V, LS Tzouvelekis, S Rossiter, E Tzelepi, FJ Angulo and JM Whichard, 2003. Imipenem resistance in a Salmonella clinical strain due to plasmid mediated class A carbapenemase KPC-2. Antimicrob. Agents Chemother., 47: 1297-300.

Sack RB, M Rahman, M Yunus and EH Khan, 1997. Antimicrobial resistance in organisms causing diarrheal disease. Clin. Infect. Dis., 24: 102-105.

Schwarz S and E Chaslus-Dancla, 2001. Use of antimicrobials in veterinary medicine and mechanisms of resistance. Vet. Res., 32: 201-225. 
Smith SM, PE Palumbo and PD Edelson, 1984. Salmonella strain resistant to multiple antibiotics: Therapeutic implications. Pediatr. Infect. Dis., 3: 455-460.

Swartz MN, 2002. Human diseases caused by foodborne pathogens of animal origin. Clin. Infect. Dis., 34: 111122.

Threlfall EJ, IS Fisher and C Berghold, 2003. Antimicrobial drug resistance in isolates of Salmonella enterica from cases of salmonellosis in humans in Europe in 2000: results of international multi-centre surveillance. Euro Surveill, 8: 41-5.

Tollefson L, SF Altekruse and ME Potter, 1997. Therapeutic antibiotics in animal feeds and antibiotic resistance. Rev. Sci. Tech., 16: 709-715.

Varma JK, K Molbak, TJ Barrett, JL Beebe, TF Jones, T Rabatsky- Ehr, KE Smith, DJ Vugia, HG Chang and FJ Angulo, 2005. Antimicrobial- resistant nontyphoidal Salmonella is associated with excess bloodstream infections and hospitalizations. J. Infect. Dis., 191: 554-561.

Wang JY, JJ Hwang, CN Hsu, LC Lin and PR Hsueh, 2006. Bacteraemia due to ciprofloxacin-resistant Salmonella enterica serotype Choleraesuis in adult patients at a university hospital in Taiwan, 1996-2004. Epidemiol. Infect., 134: 977-984.

Xia S, RS Hendriksen, Z Xie, L Huang, J Zhang, W Guo, B Xu, L Ran and FM Aarestrup, 2009. Molecular Characterization and Antimicrobial Susceptibility of Salmonella Isolates from Infections in Humans in Henan Province, China. J. Clin. Microbiol., 47: 401-409. 